\title{
Elastic analog of graphene: Dirac cones and edge states for flexural waves in thin plates
}

\author{
Daniel Torrent, ${ }^{1, *}$ Didier Mayou, ${ }^{2, \dagger}$ and José Sánchez-Dehesa ${ }^{1, \ddagger}$ \\ ${ }^{1}$ Wave Phenomena Group, Universitat Politècnica de València, Camino de vera s.n. (Edificio 7F), ES-46022 Valencia, Spain \\ ${ }^{2}$ Institut Néel, CNRS - Université Joseph Fourier, F-38042 Grenoble, France
}

(Received 31 January 2013; published 27 March 2013)

\begin{abstract}
An elastic analog of graphene is introduced and analyzed. The system consists of a honeycomb arrangement of spring-mass resonators attached to a thin elastic layer, and the propagation properties of flexural waves along it is studied. The band-structure calculation shows the presence of Dirac points near the $K$ point of the Brillouin zone. Analytical expressions are found for both Dirac frequency and velocity as a function of the resonator's parameters. Finally, the bounded modes of infinitely long ribbons of this honeycomb arrangement are analyzed. The presence of edge states, which are studied using multiple scattering theory, is shown. It is concluded that these structures can be used to control the propagation of flexural waves in thin plates.
\end{abstract}

DOI: 10.1103/PhysRevB.87.115143

PACS number(s): 43.20.+g, 41.20.Jb

\section{INTRODUCTION}

The extraordinary properties of graphene ${ }^{1-4}$ have attracted interest in the search of analogous systems for other type of waves. These extraordinary properties are mainly due to the presence in the dispersion relation of Dirac cones, ${ }^{5}$ which are points of the band structure in which the dispersion relation shows two cones that touch at the so-called Dirac point.

The search of equivalent systems for other waves started looking at two-dimensional (2D) periodic systems with Dirac. Thus, Dirac points appear in the band structure of 2D photonic ${ }^{6-10}$ and sonic ${ }^{1-13}$ crystals, showing also extraordinary propagation properties, like Zitterbewegung, ${ }^{14}$ a near-zero refraction index, ${ }^{15}$ edge states, ${ }^{16}$ extraordinary transmission, ${ }^{11,17,18}$ or one-way propagation. ${ }^{19-22}$

An elastic analog of graphene has not been fully analyzed, although the effort to create structures to control the propagation of elastic waves in 2D systems has been remarkable. Thus phononic band-gap systems for Lamb waves have been widely studied, ${ }^{23-27}$ as well as advanced refractive structures ${ }^{28-30}$ or more complex devices based on the transformation-coordinate method. ${ }^{31-33}$

This work presents an elastic analog of graphene. It consists of a thin elastic plate loaded with a honeycomb arrangement of resonators. Under the thin-plate approximation, it is shown that the dispersion relation presents Dirac cones, which have been perfectly characterized in terms of the resonator parameters. Also, edge states have been found for infinite zigzag ribbons and numerical multiple scattering simulations of finite cluster have been performed, supporting the results predicted by the band-structure calculations.

The paper is organized as follows. In Sec. II the equation of motion for the mentioned system has been solved for the case of a honeycomb arrangement of resonators, showing that Dirac cones also appear in the mentioned structure. In Sec. III these Dirac cones are analyzed, and analytical expressions for both Dirac frequency and velocity are found as a function of the resonator's parameters. Section IV is devoted to the analysis of the dispersion relation of zigzag ribbons, the structures where edge states are expected. Section V solves the multiple scattering problem of a finite cluster of resonators, and some simulations are presented validating the infinite system analysis, showing that edge states are present in finite structures and that they can be used to control the propagation of elastic waves by subwavelength units. The work is finally summarized in Sec. VI.

\section{BAND STRUCTURE}

Let us assume that an elastic plate is loaded with a periodic distribution of spring-mass resonators with force constant $k_{R}$ and mass $m_{R}$, as it is shown in Fig. 1. The arrangement is such that there are $N$ resonators per unit cell located at positions $\boldsymbol{R}_{n \alpha}=\boldsymbol{R}_{n}+\boldsymbol{R}_{\alpha}$, where $n$ runs for all the lattice vectors and $\alpha$ runs for all the resonators within the unit cell. The governing equation of motion for the plate's displacement $W_{1}$ is given by $^{26}$

$$
\left(D \nabla^{4}-\omega^{2} \rho h\right) W_{1}(\boldsymbol{r})=\sum_{\boldsymbol{R}_{n}, \alpha} f\left(\boldsymbol{R}_{n \alpha}\right) \delta\left(r-\boldsymbol{R}_{n \alpha}\right),
$$

where $\nabla^{4}$ is the biharmonic operator, $D=E h^{3} / 12\left(1-v^{2}\right)$ is the plate bending stiffness, $\rho$ is the mass density of the plate, and $h$ its thickness. The quantity $f\left(\boldsymbol{R}_{n \alpha}\right)$ is the force due to the presence of the spring-mass resonator. This force is responsible for the movement of the mass attached to the plate. Then Newton's second law is

$$
\omega^{2} m_{R \alpha} W_{2}\left(\boldsymbol{R}_{n \alpha}\right)=f\left(\boldsymbol{R}_{n \alpha}\right),
$$

$W_{2}\left(\boldsymbol{R}_{n \alpha}\right)$ being the mass displacement. Moreover, Hook's law is

$$
f\left(\boldsymbol{R}_{n \alpha}\right)=-k_{R \alpha}\left[W_{1}\left(\boldsymbol{R}_{n \alpha}\right)-W_{2}\left(\boldsymbol{R}_{n \alpha}\right)\right] .
$$

From (2) and (3) we can solve for $W_{2}\left(\boldsymbol{R}_{n \alpha}\right)$ and the force $f\left(\boldsymbol{R}_{n \alpha}\right)$ in terms of $W_{1}\left(\boldsymbol{R}_{n \alpha}\right)$. Inserting this solution in (1), the equation of motion becomes, after some algebra,

$$
\left(\nabla^{4}-\omega^{2} \frac{\rho h}{D}\right) W_{1}(\boldsymbol{r})=\sum_{\boldsymbol{R}_{n}} \sum_{\alpha} t_{\alpha} W_{1}\left(\boldsymbol{R}_{n \alpha}\right) \delta\left(r-\boldsymbol{R}_{n \alpha}\right),
$$

where the $t_{\alpha}$ is the resonator's strength, which has been defined as

$$
t_{\alpha}=\frac{m_{R \alpha}}{D} \frac{\omega_{R \alpha}^{2} \omega^{2}}{\omega_{R \alpha}^{2}-\omega^{2}},
$$




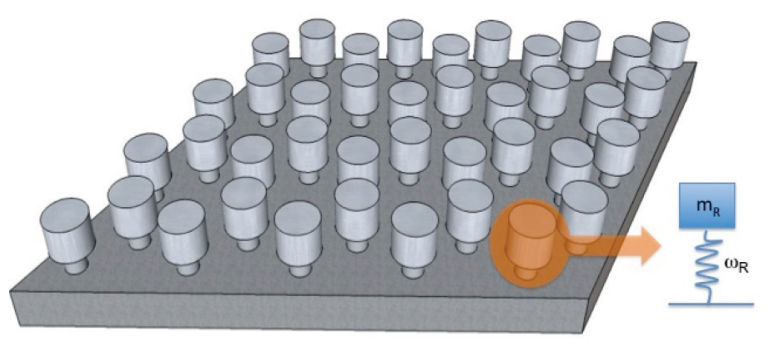

FIG. 1. (Color online) Schematic view of the system studied in this work. An array of resonators is attached to a thin elastic plate. Each resonator is modeled as a point mass attached to the plate by a spring, $m_{R}$ being its mass, and the resonant frequency $\omega_{R}$ of the mass-spring system the relevant parameters.

and $\omega_{R \alpha}=\sqrt{k_{R \alpha} / m_{R \alpha}}$ is the resonant frequency of the $\alpha$ resonator.

The periodic arrangement of resonators allows for the application of Bloch's theorem, and then $W_{1}(\boldsymbol{r})$ is expanded as

$$
W_{1}(\boldsymbol{r})=\sum_{\boldsymbol{G}} W_{\boldsymbol{G}} e^{i(\boldsymbol{K}+\boldsymbol{G}) \cdot \boldsymbol{r}},
$$

$\boldsymbol{K}$ being the Bloch wave number and $\boldsymbol{G}$ the reciprocal lattice vector. Notice that

$$
W_{1}\left(\boldsymbol{R}_{n \alpha}\right)=\sum_{\boldsymbol{G}} W_{\boldsymbol{G}} e^{i(\boldsymbol{K}+\boldsymbol{G}) \cdot \boldsymbol{R}_{n \alpha}}=e^{i \boldsymbol{K} \cdot \boldsymbol{R}_{n \alpha}} \sum_{\boldsymbol{G}} W_{\boldsymbol{G}} e^{i \boldsymbol{G} \cdot \boldsymbol{R}_{\alpha}} .
$$

Thus the equation of motion is

$$
\begin{gathered}
\sum_{\boldsymbol{G}}\left(|\boldsymbol{K}+\boldsymbol{G}|^{4} W_{\boldsymbol{G}}-\omega^{2} \frac{\rho h}{D} W_{\boldsymbol{G}}\right) e^{i(\boldsymbol{K}+\boldsymbol{G}) \cdot \boldsymbol{r}} \\
=\sum_{\boldsymbol{R}_{n}, \alpha, \boldsymbol{G}^{\prime}} t_{\alpha} e^{i \boldsymbol{K} \cdot \boldsymbol{R}_{n \alpha}} W_{\boldsymbol{G}^{\prime}} e^{i \boldsymbol{G}^{\prime} \cdot \boldsymbol{R}_{\alpha}} \delta\left(r-\boldsymbol{R}_{n \alpha}\right) .
\end{gathered}
$$

If the above equation is multiplied by $\exp [-i(\boldsymbol{K}+\boldsymbol{G}) \cdot r]$ and integrated over one unit cell, we find that

$$
\left(|\boldsymbol{K}+\boldsymbol{G}|^{4}-\omega^{2} \frac{\rho h}{D}\right) W_{\boldsymbol{G}}=\sum_{\alpha, \boldsymbol{G}^{\prime}} \frac{t_{\alpha}}{A_{c}} e^{-i \boldsymbol{G} \cdot \boldsymbol{R}_{\alpha}} W_{\boldsymbol{G}^{\prime}} e^{i \boldsymbol{G}^{\prime} \cdot \boldsymbol{R}_{\alpha}},
$$

$A_{c}$ being the area of the unit cell.

This equation defines an eigenvalue equation with eigenvectors $W_{\boldsymbol{G}}$ and eigenvalues $\omega$, from which we can obtain the dispersion relation $\omega=\omega(\boldsymbol{K})$. This procedure was employed in Ref. 26; however, in the present work, another form of Eq. (9) is required. As we will show below, the system still can be simplified.

If we define

$$
W_{\beta}=\sum_{\boldsymbol{G}^{\prime}} W_{\boldsymbol{G}^{\prime}} e^{i \boldsymbol{G}^{\prime} \cdot \boldsymbol{R}_{\beta}}
$$

and multiply Eq. (9) by $e^{i \boldsymbol{G} \cdot \boldsymbol{R}_{\beta}}$, after summing for all $\boldsymbol{G}$, we arrive at

$$
W_{\beta}=\sum_{\boldsymbol{G}} \frac{1}{|\boldsymbol{K}+\boldsymbol{G}|^{4}-\omega^{2} \rho h / D} \frac{1}{A_{c}} \sum_{\alpha} e^{i \boldsymbol{G} \cdot \boldsymbol{R}_{\alpha \beta}} t_{\alpha} W_{\alpha}
$$

or, equivalently,

$$
\sum_{\beta}\left[\delta_{\alpha \beta}-\chi_{\alpha \beta}\right] W_{\beta}=0,
$$

where

$$
\chi_{\alpha \beta}=\frac{t_{\alpha}}{A_{c}} \sum_{\boldsymbol{G}} \frac{e^{-i \boldsymbol{G} \cdot \boldsymbol{R}_{\alpha \beta}}}{|\boldsymbol{K}+\boldsymbol{G}|^{4}-\omega^{2} \rho h / D} .
$$

This quantity can be expressed in a simpler form if it is multiplied and divided by $a^{4}, a$ being the lattice constant. It is easy to show that

$$
\chi_{\alpha \beta}=\frac{m_{R \beta} a^{4}}{A_{c} D} \frac{\omega^{2}}{1-\omega^{2} / \omega_{R \beta}^{2}} \sum_{\boldsymbol{G}} \frac{e^{-i \boldsymbol{G} \cdot \boldsymbol{R}_{\alpha \beta}}}{|\boldsymbol{K}+\boldsymbol{G}|^{4} a^{4}-\omega^{2} \rho h a^{4} / D}
$$

and, by defining the normalized frequency $\Omega$ as

$$
\Omega^{2}=\omega^{2} \frac{\rho a^{2} h}{D}
$$

and the parameter $\gamma_{\beta}$ as the ratio between the mass of the resonator and that of the unit cell, that is,

$$
\gamma_{\beta}=\frac{m_{R \beta}}{\rho A_{c} h},
$$

we arrive at

$$
\chi_{\alpha \beta}=\frac{\gamma_{\beta} \Omega^{2} a^{2}}{1-\Omega^{2} / \Omega_{R \beta}^{2}} \sum_{\boldsymbol{G}} \frac{e^{-i \boldsymbol{G} \cdot \boldsymbol{R}_{\alpha \beta}}}{|\boldsymbol{K}+\boldsymbol{G}|^{4} a^{4}-\Omega^{2} a^{2}},
$$

which allows computing the frequencies $\Omega a$ of the band structure as a function of $\boldsymbol{K} a$ once the position and properties of all the resonators within the unit cell, $\gamma_{\alpha}, \Omega_{R \alpha}$ and $\boldsymbol{R}_{\alpha}$, are given.

Equation (12) defines a homogeneous system of $N$ equations, $N$ being the number of resonators per unit cell. In order to have nontrivial solutions, we need that the determinant of the matrix defining the system of equations be equal to zero. The values of $\Omega$ that for a given $\boldsymbol{K}$ makes the determinant of such a matrix equal to zero defines the band structure $\Omega=\Omega(\boldsymbol{K})$.

The band structure for a rectangular array of these resonators has been already studied in Ref. 26, where the band gaps and transmission through finite structures were analyzed. In this work we consider the honeycomb arrangement shown in Fig. 2, which is a triangular lattice with two resonators per unit cell. We have chosen this arrangement since this work is devoted to the search of an elastic analog of graphene, where carbon atoms are arranged in a honeycomb lattice.

Figure 3 shows the solution of Eq. (12) for a honeycomb arrangement of resonators, that is, a unit cell defined by the lattice vectors

$$
\begin{aligned}
& \boldsymbol{a}_{1}=a \hat{\boldsymbol{x}}, \\
& \boldsymbol{a}_{2}=a \cos \frac{\pi}{3} \hat{\boldsymbol{x}}+a \sin \frac{\pi}{3} \hat{\boldsymbol{y}},
\end{aligned}
$$

with two resonators per unit cell placed at positions

$$
\begin{aligned}
& \boldsymbol{R}_{\alpha}=\frac{a}{2 \sqrt{3}}\left(\cos \frac{\pi}{6} \hat{\boldsymbol{x}}+\sin \frac{\pi}{6} \hat{\boldsymbol{y}}\right), \\
& \boldsymbol{R}_{\beta}=-\frac{a}{2 \sqrt{3}}\left(\cos \frac{\pi}{6} \hat{\boldsymbol{x}}+\sin \frac{\pi}{6} \hat{\boldsymbol{y}}\right) .
\end{aligned}
$$




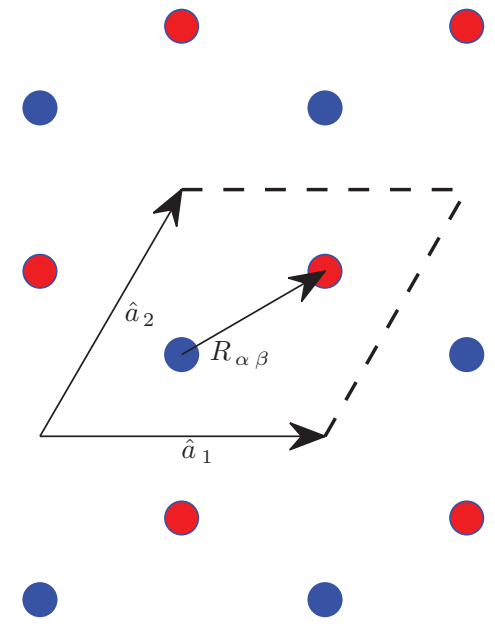

FIG. 2. (Color online) Geometry of the honeycomb arrangement of resonators. It is seen that there are two resonators per unit cell. In this analogy, every resonator is equivalent to carbon atoms in graphene.

A conical dispersion relation in Fig. 3 appears, as expected, at the $K$ points of the reduced Brillouin zone. The frequency and slope at which this cone appears are called, respectively, Dirac frequency $\Omega_{D}$ and velocity $c_{D}$.

In the next section, Eq. (12) is solved in the neighborhood of these points, and analytical expressions for both $\Omega_{D}$ and $c_{D}$ are obtained.

\section{DIRAC FREQUENCY AND VELOCITY}

The honeycomb lattice, which can be described by a triangular lattice with two resonators per unit cell, produces solutions to Eq. (12) that are obtained from the zeros of the $2 \times 2$ determinant,

$$
\left|\begin{array}{cc}
1-\chi_{\alpha \alpha} & -\chi_{\alpha \beta} \\
-\chi_{\alpha \beta}^{*} & 1-\chi_{\alpha \alpha}
\end{array}\right|=0,
$$

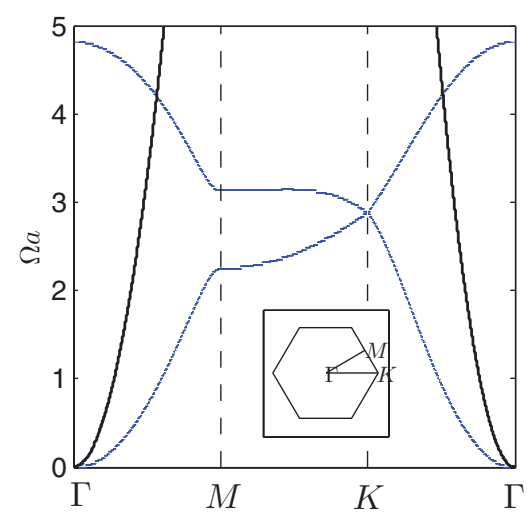

FIG. 3. (Color online) Band structure for the flexural waves propagating the system of attached resonators with the honeycomb arrangement. In this example, $\gamma_{R}=10.0$ and $\Omega_{R} a=4 \pi$. At the $K$ point the doubly degenerated conical-like dispersion relation called a Dirac cone can be seen.

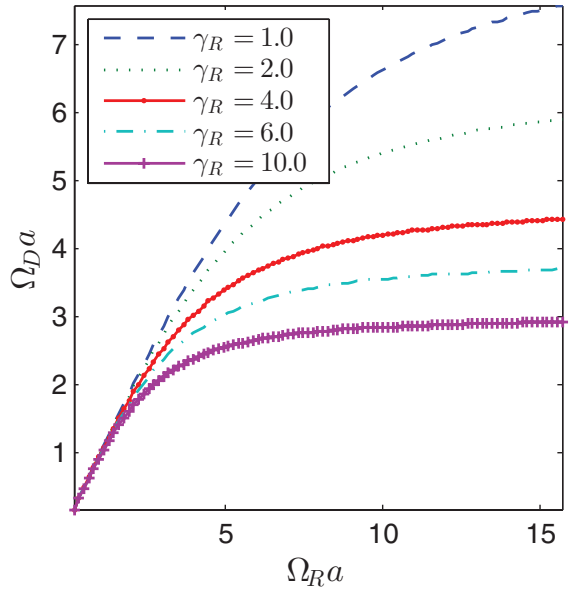

FIG. 4. (Color online) Normalized Dirac frequency (frequency of the vertex of the cone in the band structure) as a function of the normalized resonant frequency of the spring-mass system for different $\gamma_{R}$ values.

where we have used that $\boldsymbol{R}_{\alpha \beta}=-\boldsymbol{R}_{\beta \alpha}$. The solutions are then

$$
1=\chi_{\alpha \alpha} \pm\left|\chi_{\alpha \beta}\right| \text {. }
$$

The resulting band structure for a system with $\gamma_{\alpha} \equiv \gamma_{R}=$ 10.0 and $\Omega_{R \alpha} a \equiv \Omega_{R} a=4 \pi$ is shown in Fig. 3. The Dirac point appears at $\boldsymbol{K} a=\boldsymbol{K}_{D} a=4 \pi / 3$, where $\chi_{\alpha \beta}=\delta_{\alpha \beta}$, so that Eq. (21) becomes simply $1=\chi_{\alpha \alpha}$; therefore

$$
\chi_{\alpha \alpha}^{D}=\frac{\gamma_{R} \Omega_{D}^{2} a^{2}}{1-\Omega_{D}^{2} / \Omega_{R}^{2}} \sum_{\boldsymbol{G}} \frac{1}{\left|\boldsymbol{K}_{\boldsymbol{D}}+\boldsymbol{G}\right|^{4} a^{4}-\Omega_{D}^{2} a^{2}}=1
$$

solves for Dirac frequency $\Omega_{D}$ as a function of the resonator's parameters $\gamma_{R}$ and $\Omega_{R}$.

Figure 4 shows a plot of Dirac frequency as a function of the resonant frequency $\Omega_{R}$ for several values of $\gamma_{R}$. Note that Eq. (22) implies an infinite number of solutions for $\Omega_{D}$, but we keep only the lower value, since at higher frequencies the wave Eq. (1) is no longer valid.

The denominator in Eq. (22) will be dominated by the term $\left|\boldsymbol{K}_{\boldsymbol{D}}+\boldsymbol{G}\right|^{4}$ due to the fourth power of the modulus; thus this equation can be simplified if we assume that the infinite sum involved is independent of $\Omega_{D}$, that is,

$$
\sum_{\boldsymbol{G}} \frac{1}{\left|\boldsymbol{K}_{\boldsymbol{D}}+\boldsymbol{G}\right|^{4} a^{4}-\Omega_{D}^{2} a^{2}} \approx \sum_{\boldsymbol{G}} \frac{1}{\left|\boldsymbol{K}_{\boldsymbol{D}}+\boldsymbol{G}\right|^{4} a^{4}} \equiv S_{0} .
$$

Thus we can solve for $\Omega_{D}$ as

$$
\Omega_{D} a=\frac{1}{\sqrt{\gamma_{R} S_{0}+1 / \Omega_{R}^{2} a^{2}}} .
$$

From this expression it can be concluded that for $\Omega_{R} a \rightarrow \infty$ we have that $\Omega_{D} a \rightarrow\left(\gamma_{R} S_{0}\right)^{-1 / 2}$.

Figure 4 demonstrates the large degree of tunability for the Dirac frequency. Furthermore, note that the Dirac frequency can be kept constant by properly changing the pairs $\left(\Omega_{R}, \gamma_{R}\right)$; thus two different "graphenelike" media can be obtained with the same Dirac frequency.

The procedure to determine the Dirac velocity is more complex, but it is similar to that previously shown in Ref. 13 . 
The reader is referenced to the Supplementary Material of the referenced work for further details. Thus it is shown that by performing a first-order expansion around the Dirac point $\left(\Omega_{D} a, K_{D} a\right)$, we obtain that

$$
\chi_{\alpha \beta}=\delta_{\alpha \beta}\left(1+\delta \Omega a \frac{\partial \chi_{\alpha \alpha}^{D}}{\partial \Omega a}\right)+\left(1-\delta_{\alpha \beta}\right) \delta \boldsymbol{K} a \frac{\partial \chi_{\alpha \beta}^{D}}{\partial \boldsymbol{K} a} .
$$

Once the expansion coefficients of $\delta \Omega a$ and $\delta \boldsymbol{K} a$ are properly computed, the above expression is introduced into Eq. (12) and it becomes the Dirac equation for 2D massless particles, from which we can obtain the Dirac velocity $c_{D}$.

Then the expansion coefficient for $\delta \Omega a$,

$$
\frac{\partial \chi_{\alpha \alpha}^{D}}{\partial \Omega a}=\frac{\partial}{\partial \Omega a}\left[\frac{\gamma_{R} \Omega^{2} a^{2}}{1-\Omega^{2} / \Omega_{R}^{2}} \sum_{\boldsymbol{G}} \frac{1}{|\boldsymbol{K}+\boldsymbol{G}|^{4} a^{4}-\Omega^{2} a^{2}}\right],
$$

can be simplified using Eq. (22), and it can be cast in

$$
\frac{\partial \chi_{\alpha \alpha}^{D}}{\partial \Omega a}=\frac{2}{\Omega_{D}\left(1-\Omega_{D}^{2} / \Omega_{R}^{2}\right)}+\frac{2 \gamma_{R} \Omega_{D}^{3} a^{3}}{1-\Omega_{D}^{2} / \Omega_{R}^{2}} S_{\alpha \alpha},
$$

where

$$
S_{\alpha \alpha}=\sum_{\boldsymbol{G}} \frac{1}{\left(\left|\boldsymbol{K}_{\boldsymbol{D}}+\boldsymbol{G}\right|^{4} a^{4}-\Omega_{D}^{2} a^{2}\right)^{2}},
$$

which can be solved numerically.

The expansion coefficient for $\delta \boldsymbol{K} a$ is ${ }^{13}$

$$
\begin{aligned}
\frac{\partial \chi_{\alpha \beta}^{D}}{\partial \boldsymbol{K} a}= & -\frac{\gamma_{R} \Omega_{D}^{2} a^{2}}{1-\Omega_{D}^{2} / \Omega_{R}^{2}} \sum_{\boldsymbol{G}} e^{-i \boldsymbol{G} \cdot \boldsymbol{R}_{\alpha \beta}} \\
& \times \frac{\left|\boldsymbol{K}_{\boldsymbol{D}}+\boldsymbol{G}\right|^{2} a^{2}}{\left(\left|\boldsymbol{K}_{\boldsymbol{D}}+\boldsymbol{G}\right|^{4} a^{4}-\Omega_{D}^{2} a^{2}\right)^{2}}\left(\boldsymbol{K}_{\boldsymbol{D}}+\boldsymbol{G}\right) a \\
= & -\frac{2 \gamma_{R} \Omega_{D}^{2} a^{2}}{1-\Omega_{D}^{2} / \Omega_{R}^{2}} S_{\alpha \beta}(\hat{\boldsymbol{x}}+i \hat{\boldsymbol{y}}),
\end{aligned}
$$

where

$$
S_{\alpha \beta}=\sum_{\boldsymbol{G}} \frac{\left|\boldsymbol{K}_{\boldsymbol{D}}+\boldsymbol{G}\right|^{2} a^{2} e^{-i \boldsymbol{G} \cdot \boldsymbol{R}_{\alpha \beta}}}{\left(\left|\boldsymbol{K}_{\boldsymbol{D}}+\boldsymbol{G}\right|^{4} a^{4}-\Omega_{D}^{2} a^{2}\right)^{2}}(\hat{\boldsymbol{x}}-i \hat{\boldsymbol{y}}) \cdot\left(\boldsymbol{K}_{\boldsymbol{D}}+\boldsymbol{G}\right) a .
$$

After inserting these expressions into Eq. (20) it becomes

$$
\left|\begin{array}{cc}
-\delta \Omega a / c_{D} & \delta \boldsymbol{K} \cdot(\hat{\boldsymbol{x}}+i \hat{\boldsymbol{y}}) \\
\delta \boldsymbol{K} \cdot(\hat{\boldsymbol{x}}-i \hat{\boldsymbol{y}}) & -\delta \Omega a / c_{D}
\end{array}\right|=0,
$$

where the Dirac velocity is obtained from

$$
\frac{1}{c_{D}}=\frac{1}{\left|S_{\alpha \beta}\right| \gamma_{R} \Omega_{D}^{3} a^{3}}+\Omega_{D} a \frac{S_{\alpha \alpha}}{\left|S_{\alpha \beta}\right|} .
$$

This is the Dirac equation for 2D massless particles, the same equation describing electrons in graphene..$^{5}$ Equation (31) was already derived for acoustic graphene. ${ }^{13}$

Figure 5 shows $c_{D}$ as a function of $\Omega_{R}$ for several values $\gamma_{R}$. Again we can see how this parameter can be tailored with a wide range of values. Note that $c_{D}$ is nondimensional, since neither $\delta \Omega a$ nor $\delta \boldsymbol{K} a$ have dimensions.

The Dirac velocity is usually expressed relative to that of the background, but in this case the background does not

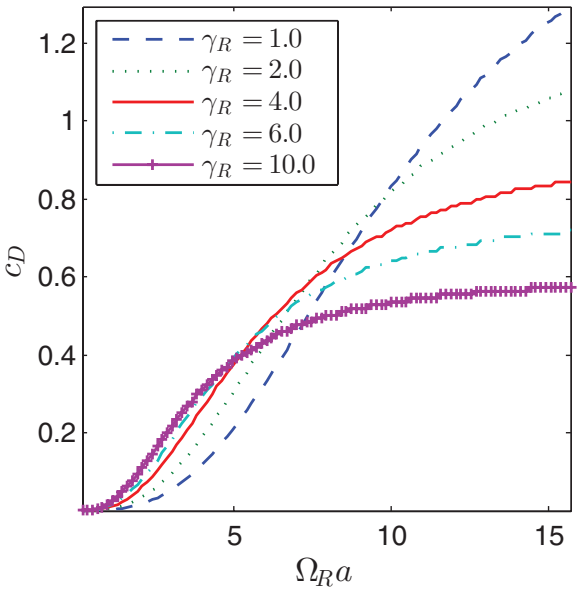

FIG. 5. (Color online) Normalized Dirac velocity as a function of the normalized resonant frequency of the spring-mass system for different $\gamma_{R}$ values.

present a linear dispersion relation, so that the comparison is not straightforward.

The physical velocity $\hat{c}_{D}=\delta \omega / \delta K$ at which Dirac waves propagate can be easily obtained from the definition of the scaled frequency $\Omega$; thus

$$
c_{D}=\frac{\delta \Omega a}{\delta K a}=\frac{\delta \omega}{\delta k} \sqrt{\frac{\rho h a^{2}}{D}},
$$

which gives

$$
\hat{c}_{D}=c_{D} \frac{h}{a} \sqrt{\frac{E}{12\left(1-v^{2}\right) \rho}} .
$$

In summary, the elastic analog of graphene can be designed by means of the resonator's properties as well as by the physical dimensions of the plate, which makes these structures of potential interest as devices for controlling elastic waves. In the next section finite slabs of elastic graphene, also called "ribbons," are studied in order to show that these ribbons, as in the electronic graphene and related analogs, present a special case of bounded states called "edge states."

\section{DISPERSION RELATION OF ZIGZAG RIBBONS}

Let us consider now the dispersion relation of finite "ribbons" of the honeycomb arrangement. The structure considered is shown schematically in Fig. 6. In this case, the ribbon is cut along what is known as a zigzag edge. The dispersion relation is computed using Eq. (13), where the unit cell is made by the resonators within the vertical rectangle in Fig. 6.

The dimensions of the unit cell are now infinite, because we are studying a waveguide, where the unit cell is defined by the rectangle $x \in[0, a], y \in(-\infty, \infty)$. Therefore in the $y$ direction we change $K_{y}+G_{y}$ by $k_{y}$, and replace the summation by an integration by performing the following transformation:

$$
\frac{1}{A_{c}} \sum_{G_{y}} \rightarrow \frac{1}{2 \pi a} \int_{-\infty}^{\infty} d k_{y} .
$$




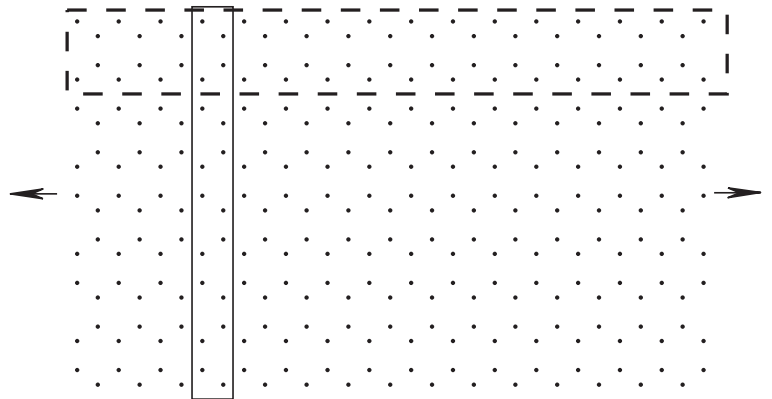

FIG. 6. Schematic view of a ribbon with zigzag edges. The left and right arrows indicate that the system is infinite along the horizontal direction ( $x$ axis). The vertical rectangle defines the unit cell that is repeated along the $x$ axis and is employed for the calculation of the dispersion relation. The horizontal dashed rectangle defines a "line." The depicted structure has five lines.

Therefore Eq. (13) becomes

$\chi_{\alpha \beta}=\frac{t_{\alpha}}{2 \pi a} \sum_{n} e^{-i 2 n \pi x_{\alpha \beta} / a} \int_{-\infty}^{\infty} \frac{e^{-i k_{y} y_{\alpha \beta}}}{|\boldsymbol{K}+\boldsymbol{G}|^{4}-\omega^{2} \rho h / D}$,

where now we have that

$$
|\boldsymbol{K}+\boldsymbol{G}|^{4}=\left[\left(K_{x}+\frac{2 n \pi}{a}\right)^{2}+k_{y}^{2}\right]^{2} .
$$

The integral in (36) can be solved using the residue theorem, leading to

$$
\chi_{\alpha \beta}=\frac{t_{\alpha}}{4 \omega \sqrt{\frac{\rho h}{D}} a} \sum_{n} e^{-i 2 n \pi x_{\alpha \beta} / a}\left[\frac{e^{-\xi_{-}\left|y_{\alpha \beta}\right|}}{\xi_{-}}-\frac{e^{-\xi_{+}\left|y_{\alpha \beta}\right|}}{\xi_{+}}\right],
$$

where

$$
\xi_{ \pm}=\sqrt{\left(K_{x}+\frac{2 n \pi}{a}\right)^{2} \pm \omega \sqrt{\frac{\rho h}{D}}}
$$

The convergence of the solution is subject to $\xi_{ \pm}$being real, which is the condition of having a guided wave in the ribbon.

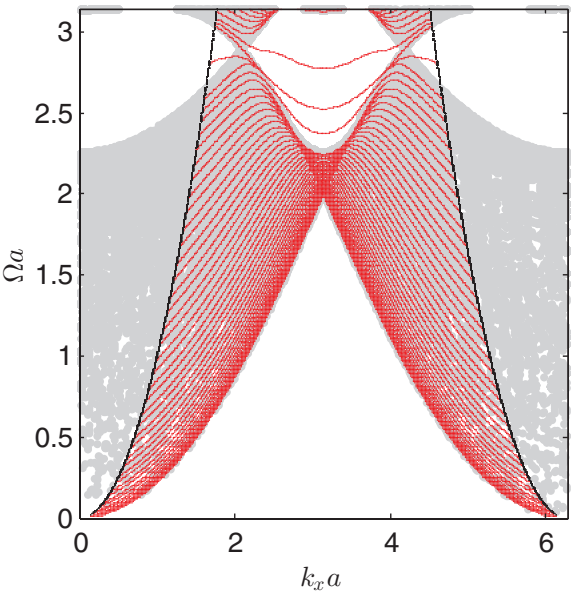

FIG. 8. (Color online) Dispersion relation of a ribbon made of ten lines with zigzag edges. The gray area is the band structure projected on the edge direction, showing clearly the difference between edge states and bulk states. The white spots in the gray regions correspond to roots not encountered by the searching algorithm due to its lack of precision.

Figure 7 shows the dispersion relation for waveguides made of $1,2,3$, and 4 lines. It is noticed that bulk modes are formed as the number of lines increases, but there are some modes, between $\Omega a=2$ and $\Omega a=3$, in which modes with flat dispersion relation appear. These are the so-called edge states.

Figure 8 shows better the difference between bulk and edge states. Here we can see the dispersion relation for a ribbon of ten lines together with the band structure of the infinite system projected along the ribbon axis (the $x$ axis). It is clear how the flat modes do not pertain to the bulk system, being bounded between the two projected Dirac points at $k_{x} a=$ $2 \pi / 3,4 \pi / 3$.

These modes are well known in electronic graphene, 3,34 as well as in both photonic ${ }^{6,7,22}$ and acoustic graphene. ${ }^{12}$ In the next section a multiple scattering method is developed for the simulations of waves propagating in ribbons under point source excitation.
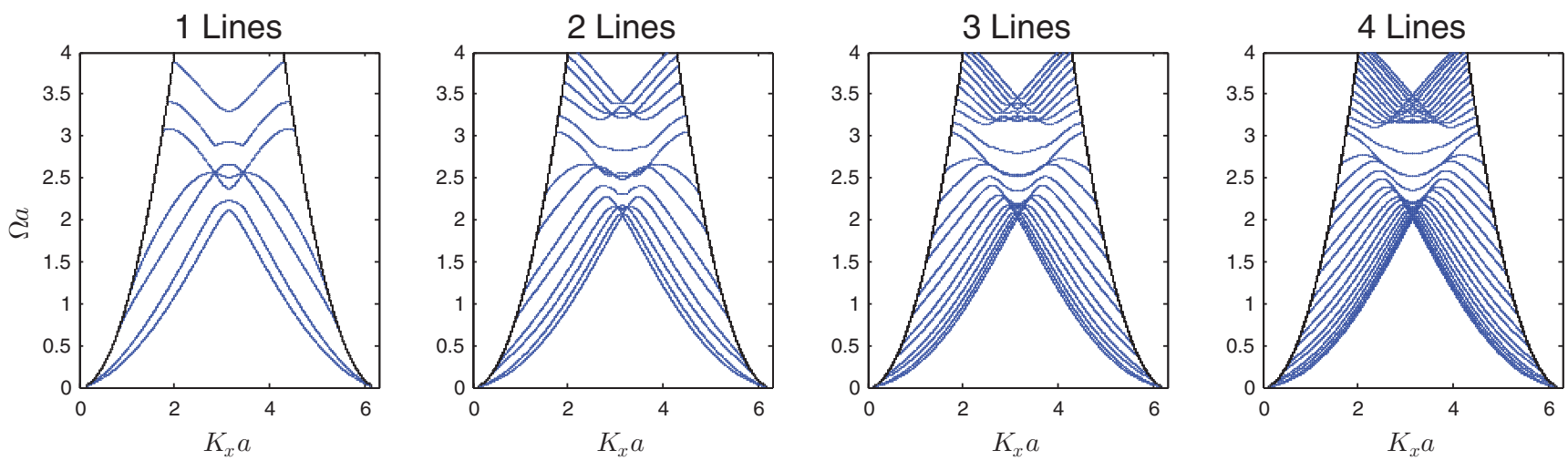

FIG. 7. (Color online) The red lines represent the dispersion relations computed for ribbons with zigzag edges with one, two, three, and four lines. The dispersion relations are limited by the free-wave dispersion relation, since outside this region the modes are leaky modes. It is clear how bulk modes are formed as we increase the number of lines but edge states appear between $\Omega a \approx 2$ and $\Omega a \approx 3$. 


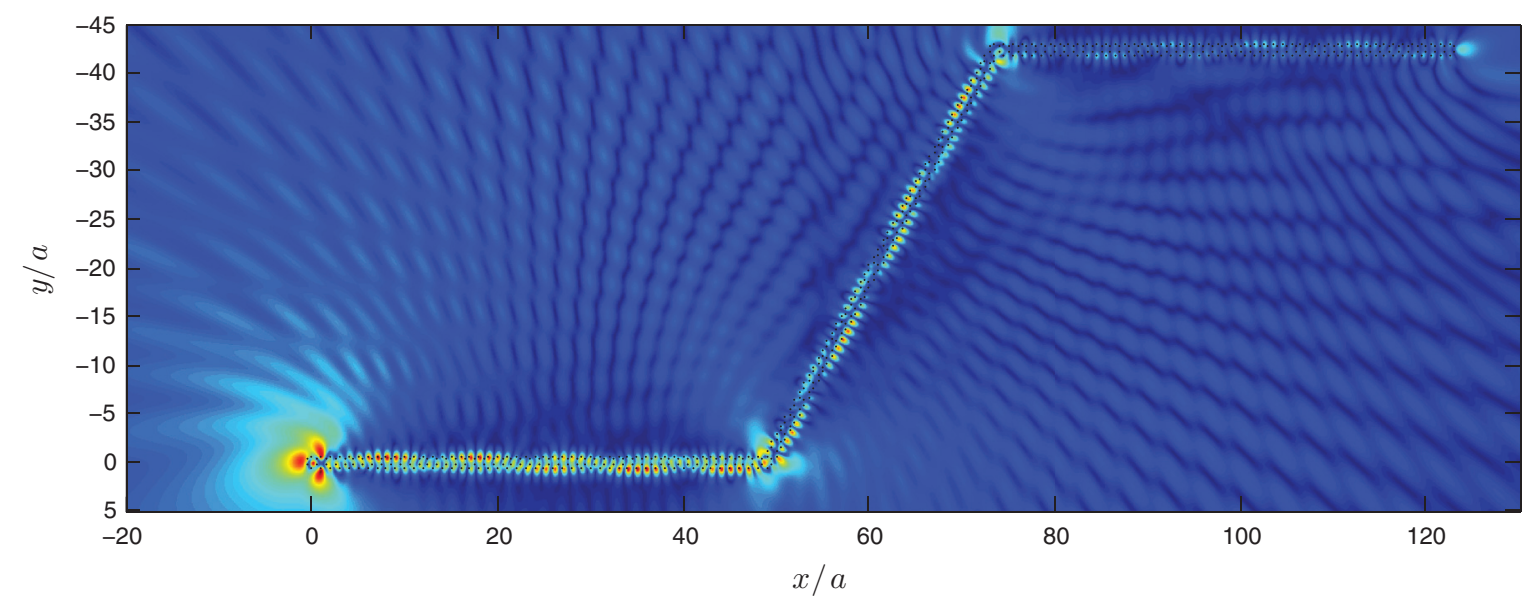

FIG. 9. (Color online) Multiple scattering simulation of an edge state excited in a single-line zigzag ribbon. The source is oscillating at a normalized frequency $\Omega a=2.6$. Note that although the state is guided along the ribbon, there are also leaky waves.

\section{MULTIPLE SCATTERING SIMULATIONS}

Let us consider now a finite cluster of resonators placed at positions $\boldsymbol{R}_{\alpha}$. The equation of motion (4) is now

$$
\left(\nabla^{4}-\omega^{2} \frac{\rho h}{D}\right) W_{1}(\boldsymbol{r})=\sum_{\alpha} t_{\alpha} W_{1}\left(\boldsymbol{R}_{\alpha}\right) \delta\left(r-\boldsymbol{R}_{\alpha}\right) .
$$

To solve the above equation we need to find a Green's function satisfying

$$
\left(\nabla^{4}-k^{4}\right) G_{0}(\boldsymbol{r})=\delta(\boldsymbol{r}),
$$

where the wave number $k$ satisfies

$$
k^{4}=\omega^{2} \frac{\rho h}{D},
$$

which has four roots, $k= \pm \sqrt{\omega}(\rho h / D)^{1 / 4}$ and $k=$ $\pm i \sqrt{\omega}(\rho h / D)^{1 / 4}$. The solutions are forward- and backwardpropagating and evanescent waves. In general, propagating waves will be described by Bessel and Hankel functions, while the evanescent waves will be described by modified Bessel and Hankel functions, since we are dealing with 2D scattering problems. The argument of all these functions always contains the modulus of the wave vector $|k|=\sqrt{\omega}(\rho h / D)^{1 / 4}$, which hereafter is named $k$.

The solution for the Green's function in Eq. (41) is

$$
G_{0}(\boldsymbol{r})=\frac{i}{8 k^{2}}\left[H_{0}(k r)+\frac{2 i}{\pi} K_{0}(k r)\right],
$$

where $H_{0}(\cdot)$ is the zeroth-order Hankel function and $K_{0}(\cdot)$ is the zeroth-order modified Bessel function of the second kind.

The problem of multiple scattering is solved by setting up a system of self-consistent equations, so that the solution for the field $W_{1}(\boldsymbol{r})$ under some incident excitation $\psi_{0}(\boldsymbol{r})$ is given by $^{35}$

$$
W_{1}(\boldsymbol{r})=\psi_{0}(\boldsymbol{r})+\sum_{\alpha} T_{\alpha} \psi_{e}\left(\boldsymbol{R}_{\alpha}\right) G_{0}\left(\boldsymbol{r}-\boldsymbol{R}_{\alpha}\right),
$$

where $\psi_{e}\left(\boldsymbol{R}_{\alpha}\right)$ is called the "external" field and is the incident field on the scatterer $\alpha$; thus

$$
\psi_{e}\left(\boldsymbol{R}_{\alpha}\right)=\psi_{0}\left(\boldsymbol{R}_{\alpha}\right)+\sum_{\beta \neq \alpha} T_{\beta} \psi_{e}\left(\boldsymbol{R}_{\beta}\right) G_{0}\left(\boldsymbol{R}_{\alpha}-\boldsymbol{R}_{\beta}\right) .
$$

The coefficients $T_{\alpha}$ can be obtained from the response of a single scatterer, since then $\psi_{e}=\psi_{0}$ and the field will be simply

$$
W_{1}(\boldsymbol{r})=\psi_{0}(\boldsymbol{r})+T_{\alpha} \psi_{0}\left(\boldsymbol{R}_{\alpha}\right) G_{0}\left(\boldsymbol{r}-\boldsymbol{R}_{\alpha}\right) .
$$

Inserting it into the wave equation we get, from the left-hand side,

$$
\begin{aligned}
& \left(\nabla^{4}-k^{4}\right)\left[\psi_{0}(\boldsymbol{r})+T_{\alpha} \psi_{0}\left(\boldsymbol{R}_{\alpha}\right) G_{0}\left(\boldsymbol{r}-\boldsymbol{R}_{\alpha}\right)\right] \\
& \quad=T_{\alpha} \psi_{0}\left(\boldsymbol{R}_{\alpha}\right) \delta\left(\boldsymbol{r}-\boldsymbol{R}_{\alpha}\right)
\end{aligned}
$$

and from the right-hand side,

$$
\begin{aligned}
& t_{\alpha} W_{1}\left(\boldsymbol{R}_{\alpha}\right) \delta\left(r-\boldsymbol{R}_{\alpha}\right) \\
& \quad=t_{\alpha}\left[\psi_{0}\left(\boldsymbol{R}_{\alpha}\right)+T_{\alpha} \psi_{0}\left(\boldsymbol{R}_{\alpha}\right) G_{0}(\mathbf{0})\right] \delta\left(\boldsymbol{r}-\boldsymbol{R}_{\alpha}\right),
\end{aligned}
$$

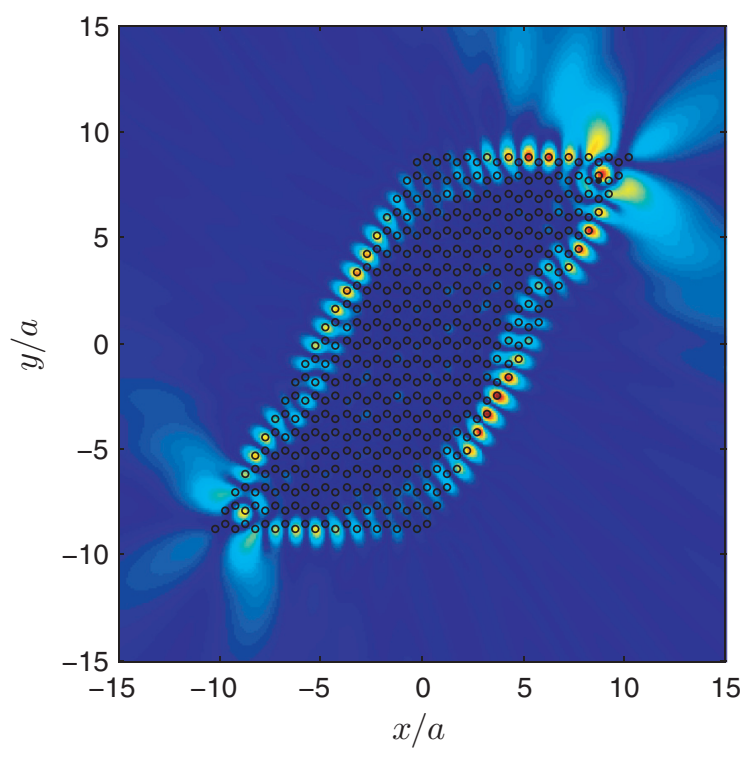

FIG. 10. (Color online) Multiple scattering simulation of a finite structure with only zigzag edges. The excitation is a point source located in the upper-right corner and oscillating at a normalized frequency $\Omega a=2.566$. The edge state is clearly observed, although a weak bulk state is also excited. 


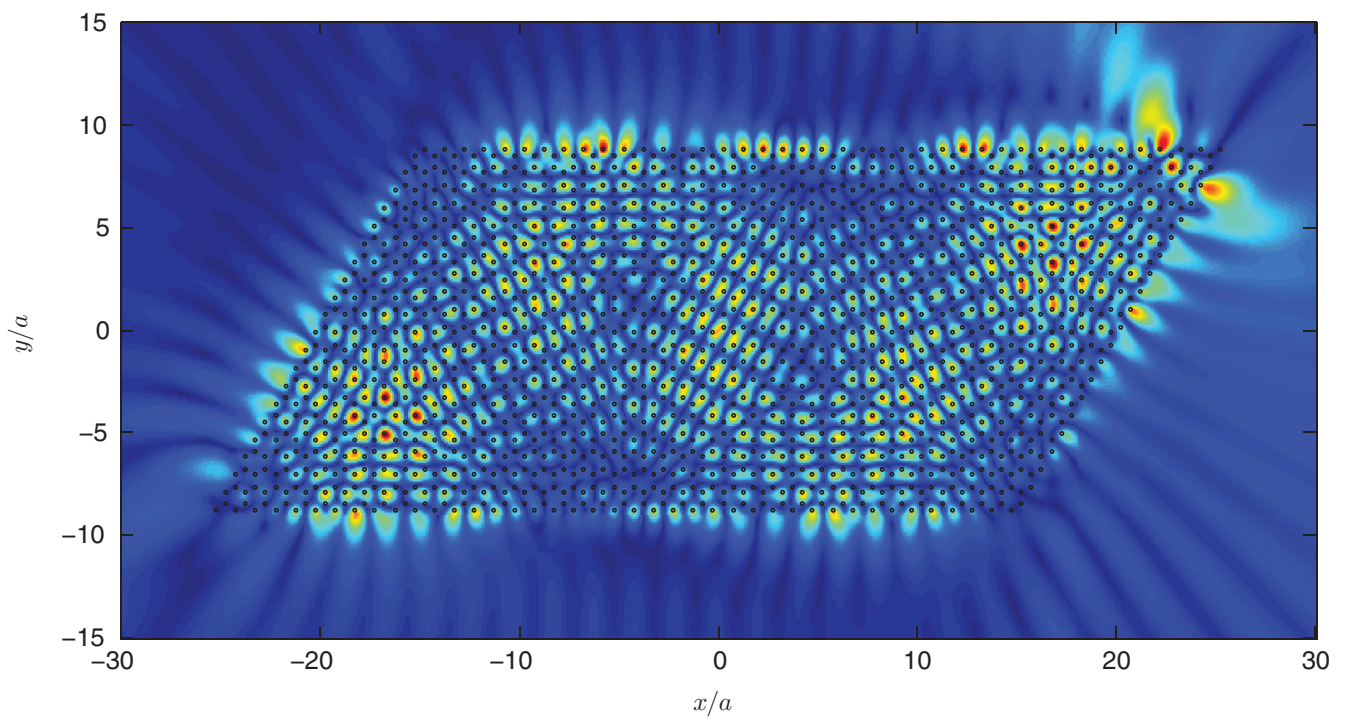

FIG. 11. (Color online) Multiple scattering simulation of a structure larger than that studied in Fig. 10 with zigzag edges. In comparison with Fig. 10, now more bulk states are excited.

which allows solving for $T_{\alpha}$ as

$$
T_{\alpha}=\frac{t_{\alpha}}{1-i t_{\alpha} /\left(8 k^{2}\right)},
$$

where we have used $G_{0}(\mathbf{0})=i /\left(8 k^{2}\right)$. Note that, unlike multiple scattering methods for point scatterers for other waves, ${ }^{35,36}$ the Green's function given by Eq. (43) is not divergent at the origin; thus no finite-size effect of the resonators needs to be added here.

Finally, we can solve for the $\psi_{e}\left(\boldsymbol{R}_{\alpha}\right)$ coefficients by solving the system of equations

$$
\sum_{\beta}\left[\delta_{\alpha \beta}-\left(1-\delta_{\alpha \beta}\right) T_{\beta} G_{0}\left(\boldsymbol{R}_{\alpha}-\boldsymbol{R}_{\beta}\right)\right] \psi_{e}\left(\boldsymbol{R}_{\beta}\right)=\psi_{0}\left(\boldsymbol{R}_{\alpha}\right) .
$$

Therefore we can completely compute the field by inserting the solutions in Eq. (45).

Figure 9 shows a multiple scattering simulation obtained by solving Eq. (50) in which a point source is placed at the origin of a one-line zigzag ribbon. The oscillation frequency of the source is set to $\Omega a=2.6$. The ribbon is twisted $60^{\circ}$ and then $0^{\circ}$ again, having a $\mathrm{z}$ shape and showing the waveguide properties of the ribbon structure. We can observe how, although some leaky waves are also excited, the field is guided along the ribbon.

Figure 10 shows a simulation in which the point source is placed at one corner of a parallelogram cluster with zigzag edges. Here the edge state nature of the excited mode is clearly observed, since the field is weak inside the cluster, and it is mainly located along the edge of the parallelogram.

Figure 11 represents a situation similar to that described in Fig. 10 but now with a larger cluster. Although the edge states still are dominant, bulk states are also excited. It shows that although edge states are present, in general the excitation of a field is made by exciting some frequency. Thus all the modes lying within this frequency will be excited as well, especially when the excitation field is a point source, which is made by a combination of all wave numbers.

\section{SUMMARY}

A two-dimensional system showing graphenelike dispersion relations has been presented. The system consists of a honeycomb arrangement of spring-mass resonators attached to a thin elastic plate, and Dirac cones have been found for the dispersion relation of flexural waves in the thin plate. These Dirac cones are characterized by a Dirac frequency, which is the vertex of the cone, and a Dirac velocity, which is the speed at which Dirac waves travel. Analytical expressions have been derived for both parameters in terms of the resonator's properties. It has been shown that both parameters can be tailored within a wide range of values.

Finite structures, also named ribbons, have been analyzed from the point of view of wave propagation, showing that they present edge states with nearly flat dispersion relations. These edge states have been studied in finite ribbons in the framework of a multiple scattering method, allowing the representation of full wave distributions and supporting the results found in the infinite and semi-infinite calculations. It has been shown that these edge states can be used to waveguide flexural waves by subwavelength units, suggesting that elastic analogs of graphene have potential applications such as devices for the control of elastic waves.

Finally, let us point out that the structures here studied are realizable in a variety of ways, for example, by attaching solid spheres to the plate or by connecting cylindrical cavities to the plate using thin channels.

\section{ACKNOWLEDGMENTS}

This work was partially supported by the Spanish Ministerio de Ciencia e Innovacion (MICINN) under Contracts No. TEC2010-19751 and No. CSD2008-66 (the CONSOLIDER program) and by the US Office of Naval Research. 
*datorma1@upv.es

†didier.mayou@grenoble.cnrs.fr

†jsdehesa@upv.es

${ }^{1}$ K. S. Novoselov, A. K. Geim, S. V. Morozov, D. Jiang, M. I. Katsnelson, I. V. Grigorieva, S. V. Dubonos, and A. A. Firsov, Nature (London) 438, 197 (2005).

${ }^{2}$ M. I. Katsnelson, K. S. Novoselov, and A. K. Geim, Nat. Phys. 2, 620 (2006).

${ }^{3}$ A. K. Geim and K. S. Novoselov, Nat. Mater. 6, 183 (2007).

${ }^{4}$ C. Beenakker, Rev. Mod. Phys. 80, 1337 (2008).

${ }^{5}$ N. Peres, Rev. Mod. Phys. 82, 2673 (2010).

${ }^{6}$ T. Ochiai and M. Onoda, Phys. Rev. B 80, 155103 (2009).

${ }^{7}$ D. Han, Y. Lai, J. Zi, Z.-Q. Zhang, and C. T. Chan, Phys. Rev. Lett. 102, 123904 (2009).

${ }^{8}$ S. Bittner, B. Dietz, M. Miski-Oglu, P. Oria Iriarte, A. Richter, and F. Schäfer, Phys. Rev. B 82, 014301 (2010).

${ }^{9}$ W. Zhong and X. Zhang, Opt. Express 19, 13738 (2011).

${ }^{10}$ K. Sakoda, Opt. Express 20, 25181 (2012).

${ }^{11}$ X. Zhang and Z. Liu, Phys. Rev. Lett. 101, 264303 (2008).

${ }^{12}$ W. Zhong and X. Zhang, Phys. Lett. A 375, 3533 (2011).

${ }^{13}$ D. Torrent and J. Sánchez-Dehesa, Phys. Rev. Lett. 108, 174301 (2012).

${ }^{14}$ X. Zhang, Phys. Rev. Lett. 100, 113903 (2008).

${ }^{15}$ X. Huang, Y. Lai, Z. H. Hang, H. Zheng, and C. T. Chan, Nat. Mater. 10, 582 (2011).

${ }^{16}$ S. R. Zandbergen and M. J. A. de Dood, Phys. Rev. Lett. 104, 043903 (2010).

${ }^{17}$ R. A. Sepkhanov, Ya. B. Bazaliy, and C. W. J. Beenakker, Phys. Rev. A 75, 063813 (2007).

${ }^{18}$ S. Bittner, B. Dietz, M. Miski-Oglu, and A. Richter, Phys. Rev. B 85, 064301 (2012).

${ }^{19}$ Z. Wang, Y. Chong, J. Joannopoulos, and M. Soljačić, Phys. Rev. Lett. 100, 013905 (2008).
${ }^{20}$ S. Raghu and F. Haldane, Phys. Rev. A 78, 033834 (2008).

${ }^{21}$ F. Haldane and S. Raghu, Phys. Rev. Lett. 100, 013904 (2008).

${ }^{22}$ Y. Poo, R.-x. Wu, Z. Lin, Y. Yang, and C. T. Chan, Phys. Rev. Lett. 106, 093903 (2011).

${ }^{23}$ J.-C. Hsu and T.-T. Wu, Phys. Rev. B 74, 144303 (2006).

${ }^{24}$ T.-T. Wu, Z.-G. Huang, T.-C. Tsai, and T.-C. Wu, Appl. Phys. Lett. 93, 111902 (2008).

${ }^{25}$ M. Oudich, Y. Li, B. M. Assouar, and Z. Hou, New J. Phys. 12, 083049 (2010)

${ }^{26}$ Y. Xiao, J. Wen, and X. Wen, J. Phys. D: Appl. Phys. 45, 195401 (2012).

${ }^{27}$ T.-T. Wu, J.-C. Hsu, and J.-H. Sun, IEEE Trans. Ultrason. Ferroelectr. Freq. Control 58, 2146 (2011).

${ }^{28}$ M. Farhat, S. Guenneau, and S. Enoch, Europhys. Lett. 91, 54003 (2010).

${ }^{29}$ M. Farhat et al., Appl. Phys. Lett. 96, 081909 (2010).

${ }^{30}$ S. Bramhavar, C. Prada, A. A. Maznev, A. G. Every, T. B. Norris, and T. W. Murray, Phys. Rev. B 83, 014106 (2011).

${ }^{31}$ M. Farhat, S. Guenneau, S. Enoch, and A. B. Movchan, Phys. Rev. B 79, 033102 (2009).

${ }^{32}$ M. Farhat, S. Guenneau, and S. Enoch, Phys. Rev. Lett. 103, 024301 (2009).

${ }^{33}$ N. Stenger, M. Wilhelm, and M. Wegener, Phys. Rev. Lett. 108, 014301 (2012).

${ }^{34}$ K. Nakada, M. Fujita, G. Dresselhaus, and M. Dresselhaus, Phys. Rev. B 54, 17954 (1996).

${ }^{35}$ L. L. Foldy, Phys. Rev. 67, 107 (1945).

${ }^{36}$ P. A. Martin, Multiple Scattering: Interaction of Time-Harmonic Waves with $N$ Obstacles (Encyclopedia of Mathematics and its Applications) (Cambridge University Press, Cambridge, UK, 2006), p. 450. 